\title{
Sylvie Lannegrand et Véronique Montémont (dir.), Résistances intérieures. Visages du conflit dans le journal personnel,
}

Academia-L'Harmattan, coll. «Au cœur des textes », 2016, 178 p.

Arnaud Genon

Nottingham Trent University

Souvent considéré comme l'espace privilégié de l'expression et de l'exploration d'un «je » intime, le journal n'en reste pas moins - ainsi que le remarquent Sylvie Lannegrand et Véronique Montémont dans leur préface - toujours intrinsèquement lié au contexte socio-historique dans lequel il a été écrit. Ainsi, le journal devient le lieu où s'inscrivent et se consignent des "résistances» dans les moments de crises politiques que sont les guerres et où naissent, lors de crises 
intérieures liées aux amours et à la sexualité, des "conflits » entre le « je » et « le discours dominant » qui l'entoure.

C'est à travers un corpus de journaux allant de la fin du XIXe siècle à l'année 2000, corpus qui regroupe des journaux classiques (George Sand, Adèle Hugo), des journaux récemment (re)découverts (Hélène Berr) ou inédits (Yves Navarre), que les chercheurs qui ont participé au présent volume tentent de répondre à la problématique suivante : comment les diaristes vivent-ils leurs engagements, leurs orientations quand ces derniers s'opposent aux circonstances historiques ou aux normes?

L'ouvrage s'organise en deux temps. Le premier, intitulé « Face à la guerre », s'intéresse aux journaux « tenus durant des conflits historiques majeurs». Le second, "Lutter pour être soi », se concentre sur les «tensions intérieures» que sont le désir amoureux et la sexualité.

\section{«Face à la guerre»}

Marion Krauthaker consacre le premier article au Journal d'un voyageur pendant la guerre de George Sand, texte peu étudié et même écarté du volume consacré aux œuvres autobiographiques de l'auteure dans La Pléiade. Tenu pendant la guerre franco-prussienne, de septembre 1870 à février 1871, ce journal naît de l'impossibilité de George Sand «d'intervenir plus publiquement sur la scène politique ». Outil cathartique, son journal n'en reste pas moins engagé contre la politique napoléonienne et donne à l'auteure l'occasion de réaffirmer ses idéaux socialistes. Si le journal s'écrit comme un «acte de guerre» qui inscrit en son sein la violence du conflit, il ne 
néglige cependant pas la sphère privée. Au contraire, "les relations avec sa famille et surtout ses petites filles représentent une source de résistance au milieu du désespoir ». En ce sens, ce texte parvient à réconcilier la femme et l'écrivain, Aurore Dupin et George Sand.

La critique consacre un second article au Journal de Mary Martin, mère de famille et veuve irlandaise qui consigna, pendant la Première Guerre mondiale, de janvier à mai 1916, le combat psychologique qu'elle mena face à l'absence de nouvelles de son fils Charlie, d'abord porté disparu puis déclaré mort des suites de ses blessures dans un camp bulgare en décembre 1915.

Françoise Simonet-Tenant s'intéresse précisément à la période de «Mai-juin 1940 dans les journaux personnels ». À partir d'un corpus de 14 textes d'écrivains publiés et reconnus (Gide, Léautaud) ou de "scripteurs inconnus», elle cherche à analyser les différentes modalités de la mise en mot de l'histoire ainsi que «le statut et les fonctions de ces textes». Les diaristes étudiés sont tous des témoins mais ils n'observent pas les événements du même endroit. Certains se trouvent sur les routes de l'exode (Roger Martin du Gard, Simone de Beauvoir) quand d'autres, comme Léautaud, n'ont pas voulu quitter la capitale. Puis il y a ceux qui se situent plus au sud (Jean Guéhenno) et voient arriver, «la rage au cœur», les Allemands dans leur ville. La confrontation des journaux fait apparaître des disparités quant à leur écriture : notes écrites sur le vif qui donnent l'impression d'assister aux événements, journal reconstitué, journal révisé parce que publié du vivant de l'écrivain (Gide), récit rétrospectif (Simone de Beauvoir) sont autant façons de relater ce moment de crise. Écrits pour «tenir le coup», pour témoigner de 
«l'extraordinaire», pour se «soulager de la violence» qui se déroule sous les yeux du diariste, ces journaux laissent souvent place à la voix des autres - presse, radio, rumeurs - comme signe de leur désarroi et d'une recherche de sens à la période qu'ils traversent. Ces journaux, conclut Françoise Simonet-Tenant, « humanisent la mémoire collective, [...] restituent la puissance de l'événement, son intensité émotionnelle et ses répercussions sur les destinées singulières ».

Après l'article de Catherine Viollet consacré au journal de Micheline Bood, lycéenne auteure d'un journal-fleuve pendant la guerre et dans lequel elle rend-compte de la vie quotidienne pendant l'occupation mais aussi de ses conflits intérieurs, et celui de Christian Lazard analysé par Cyril Grange, Véronique Montémont présente une étude qui porte sur le journal d'Hélène Berr. Elle rappelle d'abord l'histoire du manuscrit, qui aboutit au succès que l'on connaît et met ensuite en lumière les différents conflits dont le journal est porteur. Un conflit intérieur et sentimental, tout d'abord. Hélène, engagée avec Gérard, rencontre Jean Morawiecki, de qui elle tombe éperdument amoureuse. Luttant dans un premier temps contre cet amour, elle s'y abandonne ensuite, Jean l'aidant dans les épreuves qui deviennent les siennes (port de l'étoile jaune, discriminations, etc.) et devenant pour Hélène une "source de réconfort vitale». Le deuxième conflit est celui auquel elle est, avec sa famille, confrontée quotidiennement. Les lois antijuives et le port de l'étoile, l'arrestation de son père font naître chez Hélène un véritable sentiment de révolte. Se pose alors la question de partir ou de rester à Paris. Rester sera pour elle « une forme de résistance personnelle, sa manière de demeurer solidaire de tous ceux qui ont déjà été pris ». Le journal d'Hélène Berr est en même temps un éveil à lâge adulte, une catharsis lui permettant de 
mettre des mots sur ses angoisses et ses tourments, un témoignage conscient de sa portée historique, un "exercice spirituel pour faire face aux futurs douleurs ». Cet article, le dernier de la première partie, restitue de manière très juste la force et la singularité de ce remarquable journal.

\section{«Lutter pour être soi »}

Jean-Marc Hovasse nous plonge dans le journal d'Adèle Hugo sous l'angle des trois amours - Auguste Vacquerie, Auguste Clésinger et John Rose - qui le hantent. Adèle s'y imagine, selon les circonstances, en séductrice, en amante ou en épouse.

Dans «Les itinéraires d'une femme libre: Hélène Hoppenot», Hélène Gestern s'intéresse au journal de l'écrivaine, traductrice et photographe, qui tint jusqu'en 1980 un « journal-fleuve de 8000 feuillets au total » et dans lequel elle consigna des observations sur les pays qu'elle traversa avec son mari, le diplomate Henri Hoppenot (1891-1977). Ce journal, «plus extime qu'intime», laisse transparaître l'engagement féministe de la diariste qu'elle ne peut cependant exprimer publiquement. De la même façon, elle cachera à son mari la phase dépressive consécutive à la naissance de sa fille Violaine, naissance qui représente aux yeux de la jeune femme une privation de liberté. Son journal, celui d'une femme de diplomate, lui donne enfin l'occasion « de dresser des portraits piquants et lucides des microcosmes d'expatriés qu'elle côtoie ». Son regard fin et son esprit font de ce journal - dont d'autres parties seront publiées - «une ode au voyage, à la diversité et à la liberté ». 
Sylvie Lannegrand signe les deux derniers articles de l'ouvrage. L'un est consacré à Jocelyne François et, plus précisément, aux trois volumes de son journal «intermittent», l'autre au journal inédit d'Yves Navarre, dont la critique révèle deux dimensions : «la sexualité et la / le politique ». Son œuvre a souffert de n'être réduite qu'à la seule thématique homosexuelle. Présente évidemment, elle ne rend pas compte de l'ensemble du travail de l'auteur, qui soulignait qu'il était «écrivain et homosexuel » et non pas « écrivain homosexuel ». Son journal fait apparaître le parcours d'un homme engagé, fasciné par l'actualité et les faits divers. Engagé politiquement, quand il rend compte de la mort du militant de la Gauche Prolétarienne Pierre Overney, abattu par un agent de sécurité, ou pour la cause homosexuelle quand il participe, en 1975, à l'émission Les Dossiers de l'écran. Cependant, dès les années 1970, Yves Navarre prend ses distances vis-à-vis de la politique et de la cause homosexuelle, car les mouvements auxquels il participe «semblent revendiquer une différence plutôt que d'encourager l'acceptation ». S'en suivra, dans les années 1980, une véritable désillusion qui aboutira, en 1989, à son départ de la France pour le Québec. C'est dans l'écriture qu'il réinvestira finalement les valeurs qu'il ne trouvait plus « ni en politique ni dans le mouvement homosexuel », car elle seule pouvait devenir le vecteur des engagements qui étaient les siens.

Résistances intérieures. Visages du conflit dans le journal personnel ouvre encore plus les portes d'une écriture souvent, par le passé, marginalisée et minorée. Les journaux ici étudiés et mis en lumière révèlent la richesse d'un genre que l'on peut considérer, selon les mots de Véronique Montémont et de Sylvie Lannegrand, comme «l'un des observatoires les plus passionnants qui soient de l'histoire, qu'elle soit individuelle ou collective». 